\title{
VARIACIÓN DE LA ESTRUCTURA Y COMPOSICIÓN DE COMUNIDADES DE ÁRBOLES Y ARBUSTOS ENTRE TIPOS de vegetación en la Cuenca de Cuitzeo, Michoacán
}

\author{
Susana Maza-Villalobos ${ }^{1,2}$, Franceli Macedo-Santana ${ }^{3}$, Jorge Rodríguez-Velázquez ${ }^{2}$, \\ Ken Oyama ${ }^{1,2}$ y Miguel Martínez-Ramos ${ }^{2,4}$ \\ 'Escuela Nacional de Estudios Superiores, Unidad Morelia, Universidad Nacional Autónoma de México. \\ Morelia, Michoacán, México \\ ${ }^{2}$ Centro de Investigaciones en Ecosistemas, Universidad Nacional Autónoma de México. Morelia, Michoacán, México \\ ${ }^{3}$ Instituto de Investigaciones en Recursos Naturales, Universidad Michoacana de San Nicolás de Hidalgo. \\ Morelia, Michoacán, México \\ ${ }^{4}$ Autor para correspondencia: mmartinez@cieco.unam.mx
}

\begin{abstract}
Resumen: Se analizó la variación estructural de comunidades de árboles y arbustos presentes en diferentes tipos de vegetación (matorral subtropical, bosque de encino, bosque mixto, bosque de pino y bosque de oyamel) a través de gradientes de altitud y profundidad del suelo en la Cuenca de Cuitzeo, Michoacán. La cuenca cubre 4,000 $\mathrm{km}^{2}$ y un ámbito altitudinal de 1,700 a 3,420 m s.n.m. En toda la cuenca se muestrearon 50 sitios de $1,000 \mathrm{~m}^{2}$ cada uno, se registró un total de 4,941 plantas con diámetro a la altura del pecho $\geq 1 \mathrm{~cm}$, que representaron 164 especies, 88 géneros y 47 familias. Las familias con más especies fueron Asteraceae ( 30 ; $18.4 \%$ del total de especies) y Fagaceae (16; 9.8\%). Por arriba de los 2,500 m s.n.m. se encontraron los bosques de pino, oyamel y mixto (pino-encino); por debajo de 2,300 m s.n.m. se encontraron el bosque de encino y el matorral subtropical. La densidad de plantas fue semejante entre los tipos de vegetación, pero diferentes indicadores de la biomasa aumentaron con la altitud y la profundidad del suelo. El matorral subtropical y el bosque mixto fueron los más diversos en especies, mientras que el bosque de coníferas fue el menos diverso. Existió un elevado recambio de especies dentro y entre los tipos de vegetación (índice de similitud de Bray-Curtis, ámbito: 5.4-25.4\%). La composición de especies varió notablemente asociada con la altitud y la profundidad del suelo. Se ofrecen lineamientos para la conservación del importante complejo vegetal presente en la Cuenca de Cuitzeo.
\end{abstract}

Palabras claves: cuenca hidrográfica, diversidad alfa y beta, gradientes ambientales, relación altitud-vegetación, relación suelovegetación.

\begin{abstract}
This work analyzed the structural variation of tree and shrub communities present in different vegetation types (subtropical scrub, oak forest, mixed forest, pine and fir forest) across altitudinal gradients and soil depth at the Cuitzeo Basin, Michoacán. The basin covers $4,000 \mathrm{~km}^{2}$ and altitudinal range of 1,700 to $3,420 \mathrm{~m}$ a.s.l. In the entire basin we sampled 50 sites $1,000 \mathrm{~m}^{2}$ each, recording a total of 4,941 plants with a diameter at breast height $\geq 1 \mathrm{~cm}$, representing 164 species, 88 genera and 47 families. Families with more species were Asteraceae (30, 18.4\% of total species) and Fagaceae (16, 9.8\%). Above 2,500 m a.s.l. were found pine, fir and mixed (pine-oak) forests; below 2,300 m a.s.l. were found the oak forest and subtropical scrub. Plant density was similar among vegetation types but different indicators of community biomass increased with altitude and soil depth. The subtropical scrub and mixed forest were the most diverse in species and coniferous forest the less diverse. There was a high turnover of species within and among vegetation types (index of Bray-Curtis similarity, range: 5.4-25.4\%). The vegetation composition varied significantly associated with altitude and soil depth. We offer some guidelines for the conservation of the important vegetation complex present in the Cuitzeo Basin.
\end{abstract}

Key words: alfa and beta diversity, altitude-vegetation relationship, environmental gradients, hydrological watershed, soil-vegetation relationship.

$\mathbf{E}^{\mathrm{n}}$ México, la transformación de cuencas hidrográficas y a pérdida de los servicios ecosistémicos que éstas aportan, son algunos de los efectos resultantes del cambio de cobertura y uso del suelo (Masera, 2002). En los límites de los estados de Michoacán y Guanajuato se ubica la cuenca del Lago de Cuitzeo, cuya área cubre $4,000 \mathrm{~km}^{2}$ e incluye al segundo lago más extenso de México. Esta cuenca, además de ser un importante regulador del clima, cubre gran parte 
de la demanda de agua dulce de la ciudad de Morelia y de los municipios aledaños (Bravo-Espinosa et al., 2012). En las últimas décadas, el deterioro ambiental en la Cuenca de Cuitzeo ha aumentado por el cambio de uso de suelo, lo que ha resultado en una reducción de más del $50 \%$ de la cobertura histórica de la vegetación (Mendoza et al., 2004).

De acuerdo con estudios florísticos previos, en la Cuenca de Cuitzeo se han reportado entre ocho y 14 tipos de vegetación (Madrigal-Sánchez y Guridi Gómez, 2002; Bocco et al., 2012). Estudios recientes, realizados con sistemas de información geográfica a nivel de paisaje (J. Fuentes-Junco, com. pers.), indican que el matorral subtropical es el tipo de vegetación dominante, abarcando poco más del $24 \%$ de la superficie total de la cuenca; en orden decreciente le siguen el bosque mixto de pino-encino (13\%), el bosque de encino (7\%), el bosque de pino (1\%) y el bosque de oyamel $(0.1 \%)$. Producto de la conversión de ecosistemas naturales a la agricultura, los campos agropecuarios son un componente importante del paisaje en la Cuenca de Cuitzeo, los cuales representan alrededor del $40 \%$ de la cobertura del terreno (Cabrera González et al., 1999; Mendoza et al., 2010; Bocco et al., 2012).

Si bien existen algunos estudios en torno a la vegetación de la región, estos trabajos se han dirigido a la elaboración de listados florísticos (Madrigal-Sánchez y Guridi Gómez, 2002). Hasta el momento no existen estudios ecológicos que hayan analizado la variación espacial de la estructura y composición de especies de plantas de los tipos de vegetación existentes en la Cuenca de Cuitzeo. Estos estudios son requeridos para entender cómo la heterogeneidad ambiental presente en cuencas hidrográficas determina el recambio de especies y la variación en atributos estructurales de las comunidades vegetales (p. ej. Balvanera et al., 2002). A nivel de cuencas grandes, como la de Cuitzeo, los factores de clima y suelo que determinan la disponibilidad de recursos (tales como luz, agua y nutrientes minerales) y las condiciones físicas (p. ej., temperatura y humedad ambientales) que afectan el desempeño de las plantas pueden variar ampliamente con la altitud y la topografía del terreno. A través de estos gradientes ambientales se espera que la abundancia y la distribución de las especies vegetales varíen notablemente (Vázquez y Givinish, 1998).

Dada la fuerte presión del cambio de uso del suelo que ha experimentado la Cuenca de Cuitzeo (Mendoza et al., 2010), y el interés de las autoridades gubernamentales en la conservación y el manejo de esta cuenca, es urgente realizar estudios en torno a las comunidades vegetales que, más allá de describir su estructura y composición, evalúen cuáles variables ambientales determinan dichos atributos. El manejo adecuado de las cuencas hidrográficas es fundamental para el mantenimiento de los servicios ecosistémicos que son de beneficio a las poblaciones humanas (Secretaría de la Convención de Ramsar, 2010). En este sentido, el análisis ecológico de las comunidades vegetales es un elemento importante en el diseño de estrategias de aprovechamiento, restauración y conservación de la biodiversidad y los servicios que proporcionan los ecosistemas. Con esta información se coadyuva a la toma de decisiones sobre qué áreas son prioritarias para la conservación y cuáles para actividades alternativas (p. ej., extracción de productos forestales). Por otro lado, estudios de esta naturaleza representan el primer paso en el entendimiento de la historia y dinámica de las comunidades bióticas (Ashton et al., 2004).

Este estudio pretende ser una contribución al conocimiento ecológico de la vegetación presente en la Cuenca del Cuitzeo. En particular, se plantean las siguientes preguntas: ¿cuál es la estructura, la composición y la diversidad de especies de comunidades leñosas presentes en los principales tipos de vegetación de la Cuenca de Cuitzeo? y, ¿en qué grado la estructura y la composición de las comunidades estudiadas varían con la altitud y la profundidad del suelo? Finalmente, con base en los resultados se proponen algunos lineamientos de relevancia para la conservación de la vegetación de la cuenca.

\section{Materiales y métodos}

Localidad de estudio. El área de estudio comprendió la cuenca del Lago de Cuitzeo localizada en la Faja Volcánica Transmexicana (Ferrusquía-Villafranca, 1993), entre las coordenadas $19^{\circ} 30^{\prime}$ y $20^{\circ} 05^{\prime}$ latitud norte y $100^{\circ} 35^{\prime}$ y $101^{\circ} 30^{\prime}$ longitud oeste (Figura 1), abarcando una superficie de aproximadamente $4,000 \mathrm{~km}^{2}$ y un ámbito altitudinal de 1,700 a 3,420 m s.n.m. (Madrigal-Sánchez y Trujillo-García, 2001; Mendoza et al., 2010; Bocco et al., 2012). De acuerdo con la clasificación de Köppen modificado por García (1988), el clima general de esta región es $\mathrm{Cb}(w o)(w)(e) g$, que corresponde a un clima templado sub-húmedo con lluvias en verano que es fresco y largo. Sin embargo, ya que se trata de una cuenca grande, las características climáticas varían con la altitud (Leal-Nares et al., 2010). Dentro de la cuenca, existe un incremento de precipitación y un descenso de temperatura al aumentar la altitud (Leal-Nares et al., 2010); la temperatura promedio mensual en las partes altas es $10{ }^{\circ} \mathrm{C}$, de $15{ }^{\circ} \mathrm{C}$ en las partes medias y de $20^{\circ} \mathrm{C}$ en las partes bajas. La precipitación promedio anual en las partes medias es de 1,000 mm, de $600 \mathrm{~mm}$ en las partes bajas y de 1,500 mm en las partes más altas; los meses más lluviosos van de julio a septiembre, y los más secos de noviembre a abril (Mendoza et al., 2010).

Topográficamente, el $80 \%$ del área de la cuenca está compuesta de montañas y lomeríos, y sólo el $20 \%$ son terrenos planos (Mendoza et al., 2010; Bocco et al. 2012). La Cuenca de Cuitzeo es de tipo endorreica y el principal cuerpo de agua a la cual alimenta es el lago de Cuitzeo, que forma parte de la depresión lacustre Cuitzeo-Chapala (Garduño-Monroy et al., 1999). 


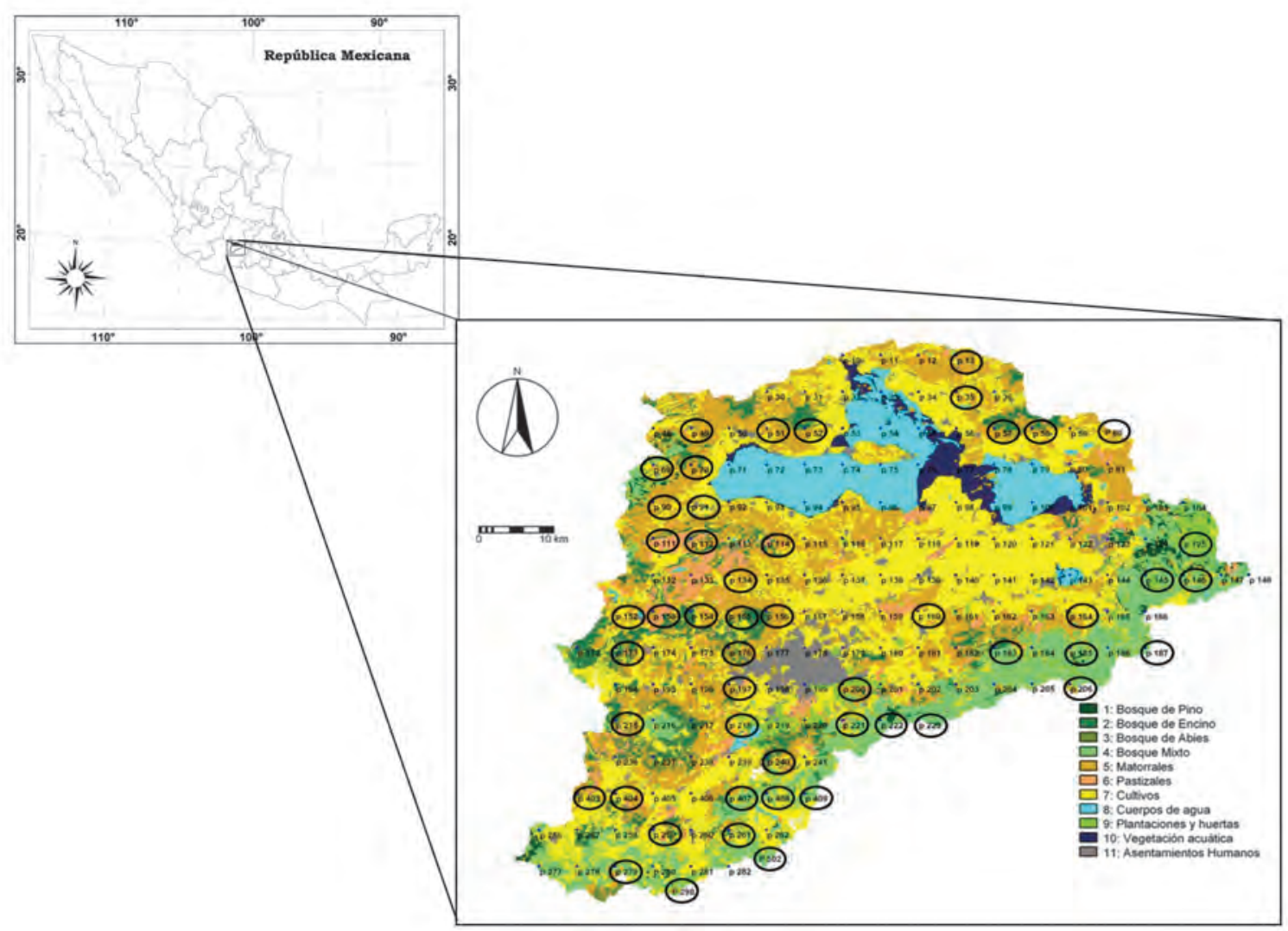

Figura 1. Mapa del área de estudio, cuenca del Lago de Cuitzeo. Los diferentes colores representan diferentes tipos de vegetación dentro de la cuenca (ver leyenda). Los puntos encerrados en óvalos indican la posición geográfica de los sitios de muestreo empleados en este estudio $(n=50)$.

Selección de los sitios de muestreo. Empleando un sistema de información geográfica (SIG; J. Fuentes-Junco, com. pers.), se generó un mapa de la superficie abarcada por la Cuenca de Cuitzeo. El mapa resultante fue reticulado en unidades de $5 \times 5 \mathrm{~km}$; se obtuvieron así 162 puntos de cruce, correspondientes a los vértices de estas unidades. De éstos, 70 puntos correspondieron a sitios con vegetación aparentemente no alterada por actividades humanas y el resto a sitios usados para fines agropecuarios, centros rurales y urbanos. De los 70 puntos, en el presente estudio se seleccionaron 50 para llevar a cabo los muestreos de la vegetación. Debido a que el área que representa cada tipo de vegetación dentro de la cuenca es diferente (J. Fuentes-Junco, com. pers.), el número de puntos de muestreo por tipo de vegetación se hizo de manera proporcional a su representación espacial relativa; es decir, de acuerdo al porcentaje de área total de la cuenca que ocupa cada tipo de vegetación. De acuerdo con la clasificación de tipos de vegetación de INEGI (2005), los 50 puntos, llamados de aquí en adelante sitios de muestreo, se distribuyeron de la siguiente manera: 24 en el matorral subtropical, diez en el bosque de encino, 13 en el bosque mixto de encino-pino, dos en el bosque de pino y uno en bosque de oyamel. Los sitios de muestreo se encontraron distribuidos en un gradiente altitudinal que varió entre 1,800 y 2,945 m s.n.m.
Muestreo de la vegetación. En cada uno de los 50 sitios se realizó un muestreo de vegetación empleando una modificación del método propuesto por Gentry (1982). En cada sitio se establecieron diez transectos de $50 \mathrm{~m}$ de largo por $2 \mathrm{~m}$ de ancho (0.01 ha), los transectos se ubicaron paralelamente a una distancia de $25 \mathrm{~m}$ entre ellos, con el fin de cubrir un área total de una hectárea. Dentro de cada transecto se registraron a todos los árboles (plantas leñosas con un sólo tallo principal) y arbustos (con varios tallos leñosos emergiendo del suelo) con un diámetro a la altura del pecho (DAP, $1.30 \mathrm{~m}$ de altura) $\geq 1 \mathrm{~cm}$ y cuya área de enraizamiento estuviese al menos 50\% dentro del transecto. Para cada una de las plantas registradas, se obtuvieron datos de altura (del nivel del suelo al ápice de la planta), cobertura de la copa (diámetro mayor y diámetro menor de copa), DAP y su identidad taxonómica (basados en colectas botánicas). La identificación taxonómica del material botánico estuvo a cargo de expertos del Centro de Investigaciones en Ecosistemas de la Universidad Nacional Autónoma de México, del Instituto de Ecología A.C. y de la Universidad Michoacana de San Nicolás de Hidalgo. Adicionalmente, con el fin de obtener un indicador del índice de área foliar por sitio, se obtuvieron mediciones del porcentaje de cobertura del dosel (porcentaje de cielo cubierto por hojas, ramas y troncos), con un densitómetro se 
obtuvieron seis mediciones: $0,10,20,30,40$ y $50 \mathrm{~m}$ de cada uno de los diez transectos del sitio, a $1 \mathrm{~m}$ de altura sobre el suelo. Cada medición consistió en el promedio de cuatro lecturas del densitómetro, cada lectura orientada a un punto cardinal (norte, este, sur, oeste); la cobertura del dosel por sitio fue el promedio de las 60 mediciones obtenidas a lo largo de los diez transectos.

Características ambientales. En cada uno de los sitios de muestreo se obtuvieron datos de altitud y profundidad del suelo. La altitud se obtuvo mediante un posicionador geográfico (GPS marca Garmin eTrex10) en el centro de cada sitio de muestreo. Para medir la profundidad del suelo se utilizó una barrena escalada en centímetros, de $1 \mathrm{~m}$ de altura y un diámetro de $2.4 \mathrm{~cm}$, la cual fue enterrada verticalmente hasta llegar al material rocoso. En cada sitio, se tomaron cinco lecturas de profundidad de suelo cada 10 metros a lo largo de cada uno de cinco transectos alternos; con las 25 mediciones obtenidas, se calculó el promedio y el error estándar de la profundidad del suelo por sitio.

Análisis de datos. Eficiencia del muestreo.- Se evaluó la eficiencia del muestreo de la vegetación realizado en toda la Cuenca de Cuitzeo por medio de la comparación del número total de especies registrado en los 50 sitios de muestreo con el número esperado de especies según curvas de acumulación de especies-área y los estimadores no paramétricos ACE, Chao-1, Jackknife y Bootstrap generados con el programa EstimateS versión 8 (Colwell, 2009). Estos índices, basados en la abundancia de las especies por sitio, cuantifican la cantidad de especies de baja frecuencia que pudieron haber sido omitidas por el muestreo (Colwell et al., 2012). Estos cálculos se llevaron a cabo empleando una matriz de datos conformada por los sitios como columnas, las especies como filas y las celdas conteniendo el número de plantas registradas por especie en cada sitio. Finalmente, se calculó el porcentaje de especies observado respecto al total pronosticado por los estimadores no paramétricos antes mencionados; estos porcentajes se usaron para evaluar la completitud (completeness) del muestreo realizado en la cuenca.

Se caracterizó la estructura y composición de las comunidades de árboles y arbustos presentes en toda la cuenca, conjuntando datos de todos los sitios muestreados y por tipo de vegetación. Para ello se cuantificaron las variables de densidad de plantas, cobertura de copas, área basal, altura máxima, porcentaje de cobertura y diversidad alfa de especies. Además, se analizó la variación en la composición de especies entre sitios y tipos de vegetación con un índice de diversidad beta de Bray-Curtis y mediante un análisis de ordenación multidimensional (NMDS, ver detalles más adelante).

Estructura de las comunidades.- La densidad de plantas se calculó como el número promedio de plantas ( \pm error estándar, e.e.) en $1,000 \mathrm{~m}^{2}$, por tipo de vegetación y por forma de crecimiento (p. ej. árboles y arbustos). La cober- tura foliar $(\mathrm{CF})$ por planta se calculó como $\mathrm{CF}=\pi^{*}\left(\mathrm{D}_{\text {mayor }}\right.$ ' $2) *\left(\mathrm{D}_{\text {menor }} / 2\right)$, donde $\mathrm{D}_{\text {mayor }}$ representa el diámetro mayor $\mathrm{y}$ $\mathrm{D}_{\text {menor }}$ el diámetro menor, suponiendo que la proyección de la copa sobre el suelo tiene una forma elipsoidal. Si el área transversal del tallo tiene una forma circular, se calculó el área basal $(\mathrm{AB})$ por tallo como $\mathrm{AB}=\pi^{*}(\mathrm{DAP} / 2)^{2}$; cuando la planta presentó más de un tallo, se sumaron las AB's de los tallos para obtener un solo valor planta. La cobertura y el $\mathrm{AB}$ de las plantas se sumaron para obtener valores por sitio. Posteriormente, se obtuvieron valores promedio $( \pm$ e.e.) de $\mathrm{CF}$ y $\mathrm{AB}$ por tipo de vegetación y por forma de crecimiento. También se obtuvieron valores promedio de la altura máxima (considerando la altura de la planta más alta registrada por sitio) y del porcentaje de cobertura del dosel (considerando los promedios de esta variable por sitio) por tipo de vegetación. Finalmente, se obtuvo un valor promedio de densidad de plantas, cobertura y área basal para toda la cuenca, calculando la media ponderada $[M(p)]$ para cada variable como $M(p)=\Sigma x_{i}^{*} c_{i}$, donde $x_{i}$ es el promedio de alguna variable estructural para el tipo de vegetación $i, c_{i}$ es la proporción de terreno cubierto por ese tipo de vegetación en la cuenca y $\Sigma$ es la suma de los productos $x_{i}{ }^{*} c_{i}$ para todos los tipos de vegetación (Smith et al., 2009). Estos valores se expresaron por hectárea multiplicando por diez el resultado de la media ponderada para cada variable.

Densidad de especies y diversidad alfa.- Se obtuvo el promedio ( \pm e.e.) de densidad de especies (número de especies en $1,000 \mathrm{~m}^{2}$ ) por tipo de vegetación y formas de crecimiento, así como la media ponderada para toda la cuenca. Además, para cada sitio de muestreo se calculó el índice de diversidad de Shannon-Wiener $\left(H^{\prime}\right), H^{\prime}=-\sum p_{i} \log \left(p_{i}\right)$ y su índice de equitatividad asociado $\left(J=H^{\prime} / H_{\max }\right)$; donde $p_{i}$ es la proporción aportada por la especie $i$ al área basal total de la comunidad en el sitio, y $H_{\max }$ es el logaritmo base $10 \mathrm{del}$ número de especies registrado por sitio (Magurran, 2004). Posteriormente, se obtuvo el valor promedio de diversidad por cada tipo de vegetación y la media ponderada para toda la cuenca. Finalmente, para cada tipo de vegetación se construyó una curva de dominancia-diversidad, la cual expresa tanto el número de especies como la equitatividad (evenness), esta última indicada por la pendiente de la curva; entre menor es la pendiente mayor es la equitatividad. Para construir esta curva, en el eje $x$ se ordenan a las especies de mayor a menor abundancia relativa y en el eje $y$ se grafica el valor de abundancia relativa (\%) de cada especie en escala logarítmica (Magurran, 2004).

Composición de especies.- Para evaluar la semejanza en la composición de especies entre los sitios se empleó el índice de similitud de Bray-Curtis $\left(S_{B}\right), S_{B}=\Xi\left(X_{i j}-X_{i k}\right) / \Xi\left(X_{i j}+\right.$ $X_{i k}$ ), donde $X_{i j}$ es el área basal de la especie $i$ en el sitio $j$ y $X_{i k}$ es el área basal de la especie $i$ en el sitio $k$. Los valores de este coeficiente van de 0 a 1,0 indica que los sitios no comparten ninguna especie y 1 indica que los sitios tienen la misma composición de especies. Se obtuvo el valor prome- 
dio y el error estándar de similitud entre sitios de un mismo y diferente tipo de vegetación. Complementariamente, se llevó a cabo un análisis de escalamiento multidimensional no métrico NMDS ("Non-metric multidimensional scaling analysis"), el cual ordenó a los sitios de muestreo a lo largo de un número reducido de dimensiones (Minchin, 1987). En el análisis de ordenación se utilizó una matriz de valores de similitud entre los sitios de muestreo pertenecientes a diferentes tipos de vegetación, cuyos elementos contuvo los valores del índice de Bray-Curtis antes mencionado. La ordenación se realizó con el programa Primer 5 (PRIMERE, 2001).

Análisis estadísticos. Para evaluar las diferencias entre los diferentes tipos de vegetación, en términos de densidad de plantas, cobertura, área basal, altura máxima, porcentaje de cobertura del dosel, densidad de especies y diversidad de especies, se realizaron análisis de varianza de una sola vía (ANOVA). Cuando fue necesario, las variables de respuesta se transformaron a una escala logarítmica (para el caso de variables de conteo) o angular (para el caso de porcentajes) para cumplir con la premisa de normalidad. Se aplicaron pruebas de Bonferroni de comparación múltiple a posterio$r i$ cuando los valores de ANOVA fueron significativos $(P$ $\leq 0.05$ ). Los sitios de bosque de oyamel y de pino se incluyeron en el tipo de vegetación denominado bosque de coníferas $(n=3)$.

Se empleó una prueba múltiple de análisis de varianza (MANOVA) para identificar a las dimensiones de la ordenación NMDS sobre los cuales existió una segregación significativa entre los tipos de vegetación. Cuando este fue el caso, se aplicaron pruebas a posteriori tipo Bonferroni para identificar a los tipos de vegetación que difirieron entre sí sobre estas dimensiones de la ordenación. Finalmente, se correlacionaron los valores de las dos dimensiones de ordenación principales con la altitud y la profundidad del suelo de los sitios, con el fin de explorar la asociación de estas dimensiones con la variación en tales factores ambientales. Todos los análisis estadísticos se realizaron empleando el programa estadístico DataDesk 6.1.

\section{Resultados}

Considerando a los 50 sitios muestreados, se registró un total de 4,895 plantas, $99.6 \%$ de este total fue identificado taxonómicamente (a nivel de especie, género o familia) y sólo el $0.4 \%$ quedó a nivel de morfo-especie; los ejemplares fueron depositados en el herbario del Centro de Investigaciones en Ecosistemas, UNAM. En total, se identificaron 87 taxones a nivel de especie, 41 a nivel de género, 20 a nivel de familia y 13 quedaron como morfo-especies. En toda la cuenca, se encontraron 71 géneros y 44 familias. Las familias más abundantes por su número de especies fueron: Asteraceae (30 especies), Fagaceae (17), Fabaceae (16) y
Pinaceae (11). El resto de familias contribuyó con siete o menos especies (Apéndice 1). Por formas de crecimiento, los árboles representaron 66\% de todas las plantas y el 57\% del total de especies registradas en toda la cuenca; el resto estuvo representado por la forma arbustiva.

Eficiencia del muestreo. La curva tendió a alcanzar una asíntota y mostró un número total de especies un poco menor a los predichos por las curvas de acumulación de especies generadas con modelos no paramétricos (Figura 2). Los estimadores no paramétricos utilizados indicaron que el muestreo de especies representó entre el 88\% (Jackknife) y el $93 \%$ (Bootstrap) del número total de especies potencialmente presentes en la cuenca (Figura 2).

Distribución altitudinal de los tipos de vegetación y profundidad del suelo. La Figura 3A muestra que el matorral subtropical se encontró en la menor altitud (por debajo de los 2,200 m s.n.m.) que los otros tipos de vegetación estudiados $\left(F_{3,46}=9.4, P \leq 0.001\right)$. El bosque de encino y el bosque mixto se encontraron en una posición altitudinal intermedia, mientras que el bosque de coníferas se ubicó en las altitudes mayores, por arriba de los 2,600 m s.n.m. El bosque mixto

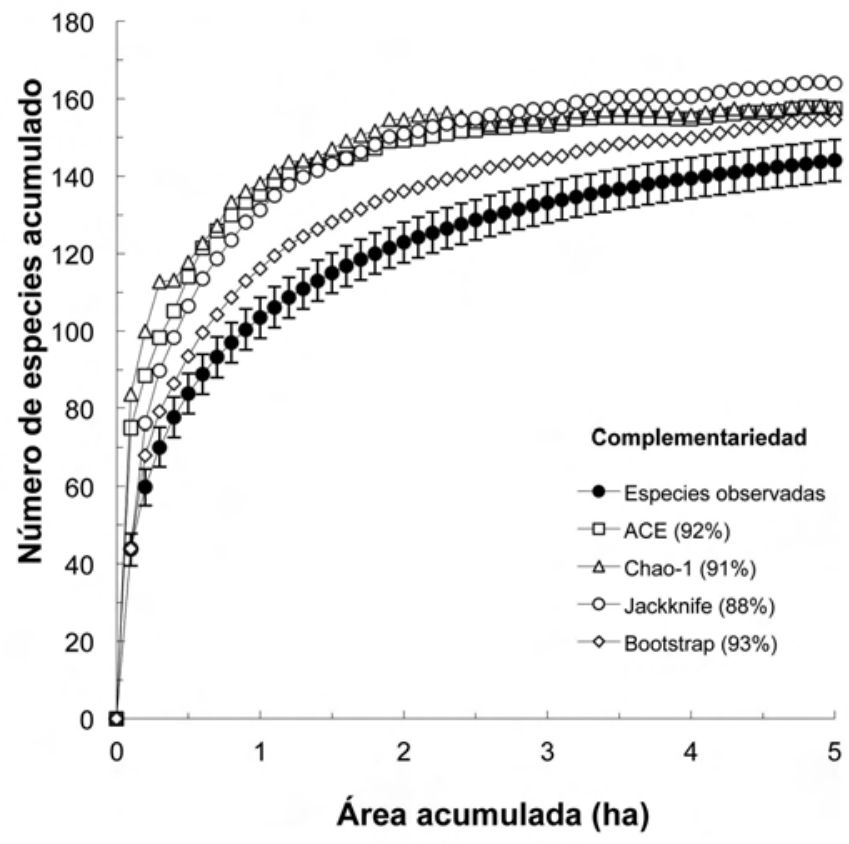

Figura 2. Curvas de acumulación de especies generadas con 50 sitios de muestreo distribuidos en la Cuenca de Cuitzeo, Michoacán, según datos observacionales (especies observadas) y estimadores no-paramétricos, basados en la abundancia de las especies muestreadas, ACE, Chao-1 (ver detalles en el texto). En la leyenda, al lado de las siglas de los estimadores, se presenta el valor de complementariedad (porcentaje del número total de especies registrado en los 50 sitios de muestreo, respecto de aquel esperado por los estimadores no-paramétricos). 

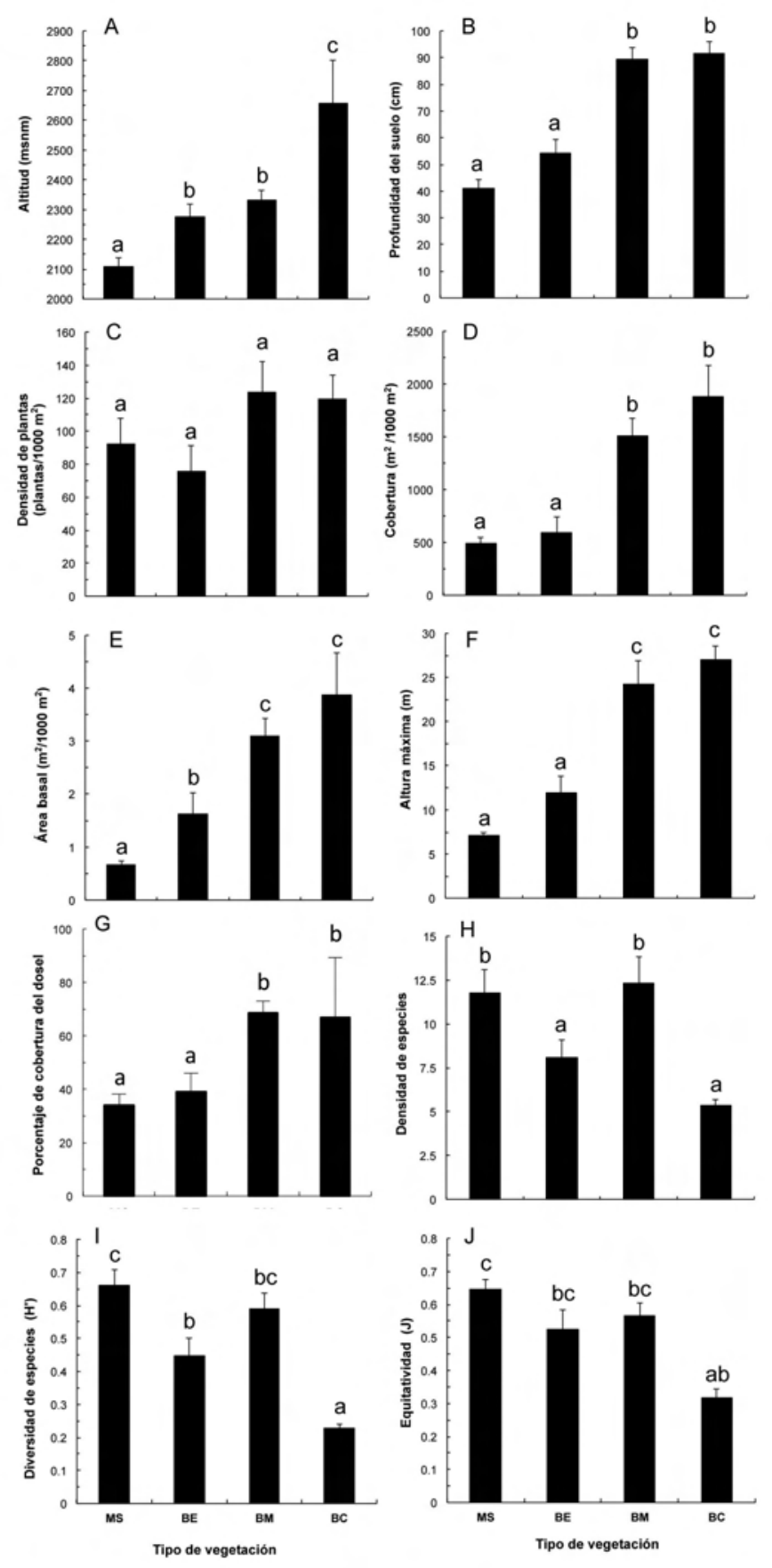

Figura 3. Variación en atributos ambientales y estructurales de comunidades leñosas entre cuatro tipos de vegetación presentes en la Cuenca de Cuitzeo, Michoacán. A) Altitud en metros sobre el nivel del mar, B) Profundidad del suelo en centímetros, C) Densidad de plantas, D) Cobertura de copas, E) Área basal, (F) Altura máxima de la vegetación, (G) Porcentaje de cobertura del dosel, (H) Densidad de especies, (I) Diversidad de especies según el índice de Shannon $\left(H^{\prime}\right)$, y (J) Equitatividad. Tipos de vegetación: $\mathrm{MS}=$ matorral subtropical, $\mathrm{BE}=$ bosque de encino, $\mathrm{BM}=$ bosque mixto de pino-encino, $\mathrm{BC}=$ bosque de coníferas. En cada panel, las columnas que no comparten letras iguales son estadísticamente diferentes a una $P \leq 0.05$. y el bosque de coníferas se presentaron en los sitios con la mayor profundidad de suelo, mientras que el bosque de encino y el matorral subtropical se presentaron en los suelos más someros $\left(F_{3,46}=19.4, P \leq 0.001\right.$; Figura 3B).

Estructura de las comunidades. En términos de la densidad de plantas, el bosque mixto y el bosque de coníferas presentaron los valores más altos en relación a los otros tipos de vegetación, pero estas diferencias no fueron significativas $\left(F_{3,46}=1.5\right.$, N.S.; Figura 3C). En contraste, la cobertura (Figura 3D) fue mayor en el bosque mixto y en el bosque de coníferas que en el bosque de encino y el matorral subtropical $\left(F_{3,46}=5.7, P=0.002\right)$. Un patrón similar se observó con la altura máxima $\left(F_{3,46}=16.3, P \leq 0.001\right.$; Figura $3 \mathrm{~F})$ y el porcentaje de cobertura del dosel $\left(F_{3,46}=4.8, P=\right.$ 0.005 ; Figura $3 \mathrm{G})$, mientras que el área basal aumentó gradualmente desde el matorral subtropical hasta el bosque de coníferas $\left(F_{3,46}=8.5, P<0.001\right.$; Figura $\left.3 \mathrm{E}\right)$. El promedio ponderado de densidad de plantas para toda la cuenca fue de 988.2 arbustos y árboles (DAP $\geq 1 \mathrm{~cm}$ ) por hectárea, con una cobertura de $8,593 \mathrm{~m}^{2}$ por hectárea y un área basal de $16.7 \mathrm{~m}^{2}$ por hectárea.

La contribución relativa de las formas de crecimiento de árboles y arbustos a los valores totales de densidad de plantas, cobertura, área basal y densidad de especies, varió entre los tipos de vegetación (Figura 4). En el matorral subtropical y el bosque de encino los arbustos representaron una proporción importante de la densidad de plantas y especies (35$45 \%$; Figura 4A). En los demás tipos de vegetación la contribución de los árboles fue preponderante sobre aquella de los arbustos en todas las variables estructurales analizadas.

En términos de la densidad $\left(F_{3,46}=2.9, P=0.05\right)$ y diversidad de especies $\left(F_{3,46}=5.7, P=0.002\right)$, el matorral subtropical y el bosque mixto mostraron los valores más altos, sobre todo respecto al bosque de coníferas (Figura 3H, I). De la misma manera, mientras que el matorral subtropical exhibió la equitatividad más alta, el bosque de coníferas mostró la equitatividad más baja $\left(F_{3,46}=5.1, P=0.004\right.$; Figura $\left.3 \mathrm{~J}\right)$. Las curvas de dominancia-diversidad mostraron notables diferencias estructurales entre los tipos de vegetación (Figura 5). Si bien todas las curvas se ajustaron muy bien a un modelo de la forma $\ln (Y)=\ln (a)-b X$ (donde $Y$ es el porcentaje del área basal de la especie $i, X$ es el rango de esa especie, $a$ es la ordenada al origen y $b$ la pendiente de la curva; en todos los casos $R^{2}>0.92, P<0.01$ ), el bosque de pino y el bosque de oyamel mostraron una fuerte jerarquía en la dominancia de las especies, con un bajo número de especies y una baja equitatividad (valores de $b$ muy bajos: -1.20 y -1.67 , respectivamente). El bosque de oyamel estuvo dominado fuertemente por Abies religiosa (representando $81 \%$ del área basal total) y el bosque de pino por Pinus oocarpa (48\%) y Pinus sp. (39\%). El bosque de encino mostró una diversidad intermedia; estuvo dominado por Quercus castanea $(36 \%), Q$. deserticola $(26 \%)$ y $Q$. laeta $(7 \%)$ y mostró mayor equitatividad (-0.76). El bosque mixto y el matorral 
A

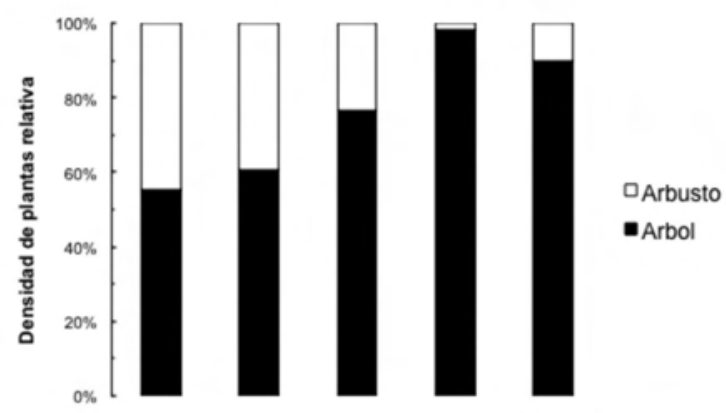

C

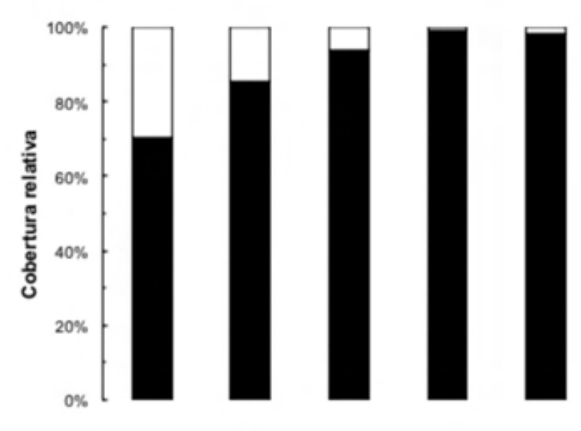

D

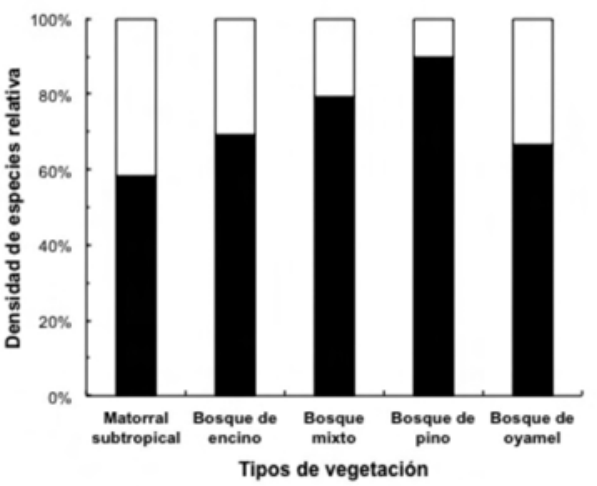

Figura 4. Contribución porcentual de árboles y arbustos a la estructura de comunidades de árboles y arbustos presentes en diferentes tipos de vegetación de la Cuenca de Cuitzeo, Michoacán. A) Densidad de plantas, B) Cobertura de copas, C) Área basal y (D) Densidad de especies.

subtropical representaron los tipos de vegetación más diversos, con mayor número de especies y la mayor equitatividad (-0.53 y -0.42 , respectivamente). En el bosque mixto ocho especies aportaron el $80 \%$ del área basal total mientras que en el matorral subtropical 19 especies cubrieron este porcentaje. Las cinco especies dominantes en el bosque mixto fueron Pinus oocarpa (26\%), P. teocote (19\%), P. leiophylla (11\%), P. michoacana (10\%) y Quercus crassifolia (4\%). En el matorral subtropical las cinco especies dominantes fueron Ipomoea arborescens (24\%), Quercus deserticola (11\%), Acacia pennatula (8\%), Eysenhardtia polystachya (4\%) y Acacia farnesiana (3\%).

Composición de especies. De manera general, el índice de similitud de Bray-Curtis mostró valores muy bajos de si- militud de especies dentro y entre los diferentes tipos de vegetación, lo que significa un notable recambio de la composición florística entre sitios y la existencia de una alta diversidad beta en la Cuenca de Cuitzeo (Cuadro 1). Con excepción del bosque de coníferas, existió mayor similitud de especies entre sitios de un mismo tipo de vegetación que entre sitios de diferente tipo de vegetación. Los tipos de vegetación con mayor similitud fueron el matorral subtropical y el bosque de encino (12.9\%), entre el bosque de encino y el bosque mixto (13.9\%) y entre el bosque mixto y el bosque de coníferas $(13.2 \%)$.

El análisis de ordenación (NMDS) corroboró los resultados anteriores. Se detectaron tres grandes grupos de sitios estadísticamente diferentes: aquellos correspondientes al matorral subtropical, el bosque de encino y la combinación

Cuadro 1. Valores promedios del índice de similitud de especies de Bray-Curtis entre cuatro tipos de vegetación leñosa de la cuenca de Cuitzeo, Michoacán. Los valores corresponden al promedio $( \pm$ e.e.) de similitud entre pares de sitios de un mismo y diferente tipo de vegetación.

\begin{tabular}{lccc}
\hline & $\begin{array}{c}\text { Matorral } \\
\text { Subtropical }\end{array}$ & $\begin{array}{c}\text { Bosque } \\
\text { de encino }\end{array}$ & $\begin{array}{c}\text { Bosque } \\
\text { mixto }\end{array}$ \\
\hline Matorral subtropical & $17.5 \pm 0.8$ & & \\
Bosque de encino & $12.9 \pm 0.6$ & $21.8 \pm 2.6$ & $30.2 \pm 1.6$ \\
Bosque mixto & $10.6 \pm 0.7$ & $13.9 \pm 1.0$ & $13.2 \pm 1.6$ \\
Bosque de coníferas & $2.7 \pm 0.7$ & $4.2 \pm 0.7$ & $11.8 \pm 11.8$ \\
\hline
\end{tabular}




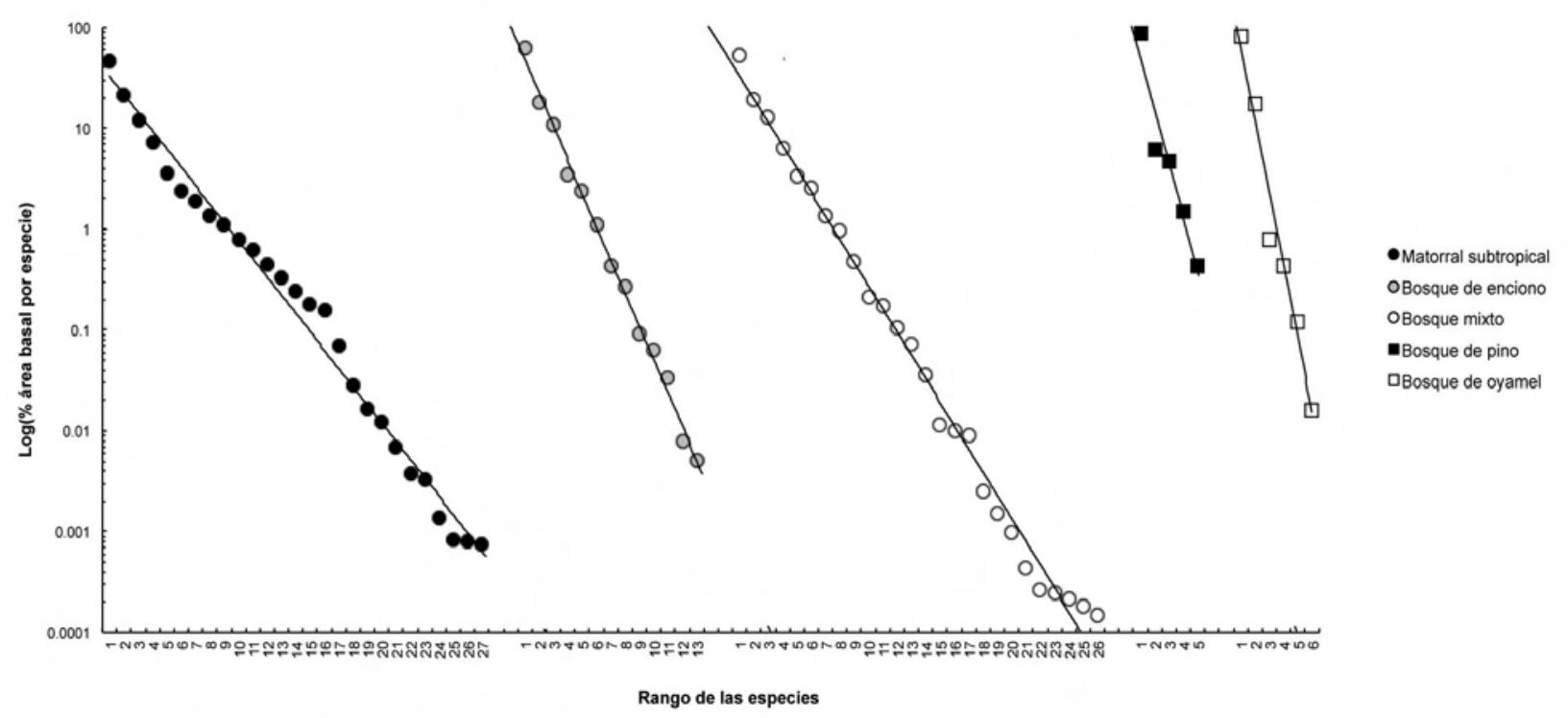

Figura 5. Curvas de dominancia-diversidad de los cinco tipos de vegetación estudiados en la Cuenca de Cuitzeo, Michoacán. En el eje $x$ se ordenan a las especies de la más a la menos abundante; en el eje y se indica la abundancia relativa de cada especie en escala logarítmica (base 10).

de sitios del bosque mixto y del bosque de coníferas (estrés: 0.015; Figura 5). El MANOVA $\left(F_{3,45}=26.1, P<0.0001\right.$; Figura 6) mostró diferencias significativas sobre la dimensión 1 y la dimensión 3 de la ordenación. Sobre la dimensión 1, el bosque mixto (más el bosque de coníferas) fue diferente al matorral subtropical $(P<0.05)$ y el bosque de encino, el cual se ubicó como un sistema transicional entre estos dos tipos de vegetación. Sobre la dimensión 3, el matorral subtropical fue diferente al bosque de encino y éste último fue diferente del bosque mixto $(P<0.05)$. La dimensión 1 del NMDS se correlacionó positivamente con la profundidad del suelo ( $r=0.79, P=0.0007)$ y con la altitud $(r=0.64, P$ $=0.0008)$, mientras que la dimensión 3 no se correlacionó con ninguna de estas variables. De esta manera se encontró que el matorral subtropical se distribuye en altitudes bajas y suelos someros, el bosque de encino en altitudes medias, mientras que el bosque mixto, el bosque de pino y el bosque de oyamel en las altitudes mayores con los suelos más profundos (Figura 3).

\section{Discusión}

La presencia de diferentes tipos de vegetación y la variación en la extensión que éstos abarcan dentro de la Cuenca de Cuitzeo expresan la heterogeneidad ambiental presente en este espacio geográfico. Tal heterogeneidad es producida en gran medida por la variación del clima y de los suelos a lo largo de un gradiente altitudinal de más de 1,700 metros (1,700 a 3,420 m s.n.m). Aun cuando esta cuenca se ubica dentro de la provincia biogeográfica Neotropical (Espinosa y Ocegueda, 2008), a través de esta variación altitudinal se encuentran especies provenientes de diversas regiones fitogeográficas (Rzedowski, 1978, 1991; Cué-Bär et al., 2006). En los sitios de estudio se encontraron en las partes más altas, templadas y húmedas de la cuenca, a especies de afinidad Neártica, tales como aquellas de los géneros Abies, Pinus y Quercus. En las partes más bajas, cálidas y secas, se encontraron a especies de afinidad Neotropical de los géneros Bursera, Cedrela y Ceiba, así como especies que habitan ambientes semiáridos, incluyendo aquellas de los géneros Acacia y Albizia, lo cual concuerda con lo establecido por Rzedowski $(1978,1991)$ y Espinosa y Ocegueda (2008).

Las familias con mayor número de especies encontradas en la cuenca fueron Asteraceae y Fagaceae. Este resultado coincide con lo reportado en otros trabajos para el estado de Michoacán (Cué-Bär et al., 2006), donde tales familias fueron las más ricas en especies. México es uno de los principales centros de diversificación de la familia Asteraceae, por lo que cuenta con el mayor número de especies en el país (Rzedowski, 1991; Villaseñor, 2003); Michoacán se ubica como el quinto estado del país con el mayor número de especies de la familia Asteraceae (Villaseñor, 1993). El género Quercus fue el más rico en especies (17) en la Cuenca de Cuitzeo, en concordancia con el hecho de que México es uno de los dos centros mundiales de diversidad de este género (Valencia-A., 2004). Asimismo, se encontró que la familia Fabaceae es una de las más representativas taxonómicamente (16 especies) en la cuenca. Lo anterior concuerda con lo reportado con otros trabajos realizados en el estado de Michoacán (Cué-Bär et al., 2006).

Estructura de las comunidades. Los resultados muestran que los tipos de vegetación encontrados en la parte baja de la 
cuenca (matorral subtropical y el bosque de encino) poseen menor cobertura, área basal, altura máxima y menor índice de área foliar; es decir, menor biomasa, que aquellos ubicados en la parte alta (bosque mixto y bosque de coníferas; Figura 3). Esto puede deberse, en parte, a que en las zonas bajas de la cuenca el suelo es somero, presumiblemente con una baja cantidad de materia orgánica, comparado con aquel encontrado en las zonas altas (Fig. 3B). Se sabe que la cantidad de nutrientes disponibles en el suelo está positivamente relacionada con la cantidad de materia orgánica (Likens et al., 1967). Sin embargo, aunque la relación positiva entre la cobertura foliar y la profundidad del suelo $\left(R^{2}=0.48 ; P\right.$ $<0.0001)$, así como aquella entre el área basal y la profundidad del suelo $\left(R^{2}=0.57 ; P<0.0001\right)$ pueden apoyar este hipótesis, es necesario hacer estudios más específicos que evalúen otros factores que influyen en la cantidad y la disponibilidad de nutrientes en el suelo de estos sitios. Otro factor que puede explicar la mayor biomasa en las partes altas de la cuenca, es el hecho de que la precipitación anual (y por lo tanto la disponibilidad de agua), es dos veces mayor en las partes altas $(1,403 \mathrm{~mm})$ que en las bajas $(683 \mathrm{~mm}$; LealNares et al., 2010).

Los menores valores de cobertura y área basal encontrados para el matorral subtropical se asocia con la elevada abundancia de arbustos (61\% del total de registros) presentes en ese tipo de vegetación; en general, los arbustos tienen tallos y copas de menor tamaño que los árboles. Además, una gran proporción (88\%) de los árboles y arbustos registrados en ese tipo de vegetación tuvieron tamaños diametrales pequeños (entre $1 \mathrm{a} 15 \mathrm{~cm}$ de DAP). La dominancia de las formas leñosas con tallos delgados en el matorral subtropical de la región de estudio ha hecho pensar a algunos autores que este tipo de vegetación representa en realidad estados sucesionales del bosque tropical caducifolio, que probablemente existía en la región, resultantes de perturbaciones antrópicas (Rzedowski y Calderón, 1989).

Formas de crecimiento. A nivel de toda la cuenca, los árboles dominaron sobre los arbustos en términos de densidad de plantas, área basal, cobertura, densidad y diversidad de

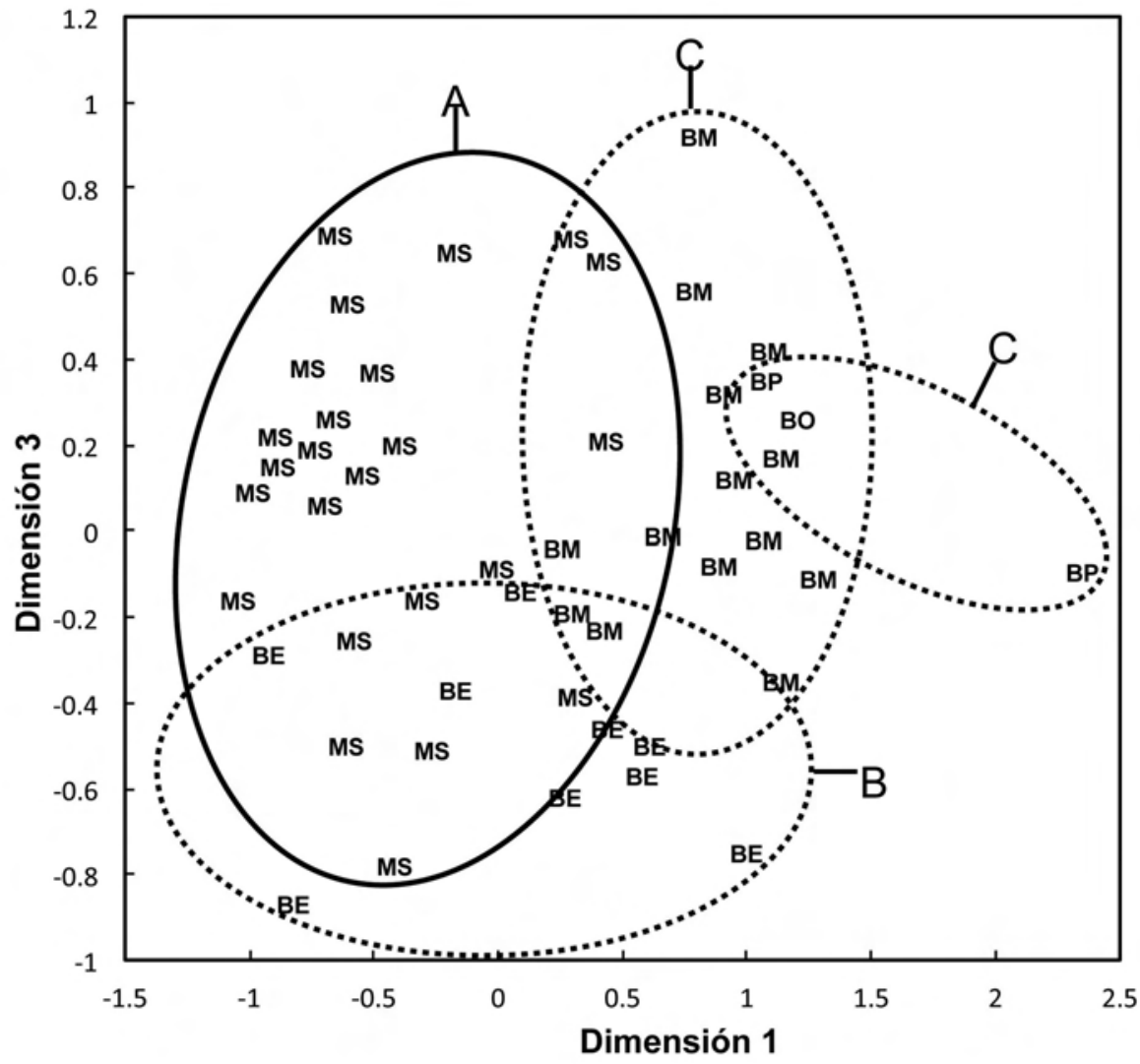

Figura 6. Escalamiento multidimensional no-métrico (NMDS, por sus siglas en inglés) de los 50 sitios de muestreo en la cuenca del lago de Cuitzeo. La posición de cada sitio en la ordenación está indicada por las siglas: MS) matorral subtropical, BE) bosque de encino, BM) bosque mixto, BP) bosque de pino y BO) bosque de Oyamel. Las elipses encierran a los sitios de un mismo tipo de vegetación: línea continua, matorral subtropical; línea puntuada, bosque de encino; línea en rayas, bosque mixto; línea en puntos y rayas, bosque de coníferas. Elipses con letras diferentes indican diferencias significativas entre los tipos de vegetación sobre la dimensión 1 de la ordenación $(P<$ 0.05). La dimensión 1 se correlacionó significativamente $(P<0.05)$ con la altitud y la profundidad del suelo, ambas variables aumentan de izquierda a derecha del eje de esa dimensión. 
especies. Sin embargo, la proporción de árboles y arbustos varió en los diferentes tipos de vegetación. En la parte baja de la cuenca, en particular en el matorral subtropical, más del 50\% de la densidad de plantas y de especies estuvo representada por arbustos, mientras que en la cuenca alta los árboles fueron estructural y taxonómicamente dominantes. Estos resultados confirman que la abundancia y el número de especies arbustivas decrece con la altitud, como se ha observado en otros estudios realizados en cuencas semejantes a la Cuenca de Cuitzeo (Encina Domínguez et al., 2009). Los arbustos están mejor adaptados a sitios con mayor radiación solar y temperatura elevadas (Grime, 1982). En bosques maduros, la baja radiación solar imperante en el sotobosque puede limitar el desarrollo de especies arbustivas que, por lo general, se caracterizan por ser heliófilas (Grime, 1982).

Diversidad de especies. Los tipos de vegetación más diversos fueron el matorral subtropical y el bosque mixto, donde se encontró el mayor número de especies y una mayor equitatividad en la dominancia de especies. Otros estudios (Madrigal Sánchez, 1995; Cabrera González et al., 1999; Cué-Bär et al., 2006) indicaron previamente que el matorral subtropical era el más diverso de la región de estudio. Sin embargo, el presente estudio muestra que el bosque mixto es similar en diversidad al matorral subtropical. Los bosques de pino y de oyamel fueron los menos diversos, los cuales estuvieron dominados fuertemente por contadas especies de coníferas. Las bajas temperaturas y heladas prevalecientes durante el invierno a altitudes mayores de $2,500 \mathrm{~m}$ en la cuenca, limitan el desarrollo de especies tropicales y favorecen a las especies de afinidad Neártica. Además, como resultado de la elevada acumulación de hojarasca (RochaLoredo y Ramírez-Marcial, 2009), los bosques de coníferas están propensos a incendios naturales. La corteza gruesa, característica de las coníferas, funciona como un aislante de los tejidos vivos ante el fuego y los conos serotinos que contienen a las semillas de las coníferas requieren de altas temperaturas, generadas por el fuego, para abrirse y dispersar las semillas (Lamont et al., 1991). Pocas especies leñosas de angiospermas han desarrollado estrategias de resistencia al fuego, lo que favorece la mono-dominancia de tales especies en sistemas sujetos a este tipo de disturbio.

Composición de especies. El índice de Bray-Curtis y el análisis de ordenación (NMDS) mostraron la existencia de un notable reemplazamiento de especies a través de las comunidades vegetales presentes en la cuenca. Tal reemplazamiento se encontró asociado con el gradiente altitudinal y el gradiente de profundidad de suelo. El análisis de ordenación separó completamente a los tipos de vegetación de las partes altas de la cuenca (> 2,500 m s.n.m., bosques de oyamel, de pino y mixtos) de aquellas de la parte baja $(<2,300 \mathrm{~m}$ s.n.m., matorral subtropical y bosques de encino).

Tal diversidad beta sugiere la presencia de muchas espe- cies con hábitats diferenciados. Las especies arbóreas que se desarrollan sólo en las partes altas con climas templados y heladas en invierno (oyameles, pinos y algunos encinos) son perennifolias con cortezas gruesas. Estas cortezas favorecen el flujo de la savia por el floema aún en tiempos de heladas. El nivel de xilol y fructosa presente en las células de las coníferas aumenta en la época de invierno, lo que impide el congelamiento celular (Strasburger et al., 1960). Por otro lado, la mayoría de las semillas, como las de los encinos, presentan una testa dura que protege al embrión de las temperaturas bajas (Rzedowski y Rzedowski, 2005). En las partes más bajas y cálidas de la cuenca se encontraron especies adaptadas a condiciones de baja disponibilidad de agua y elevados niveles de estrés térmico. Tales plantas presentan estrategias funcionales (tales como hojas reducidas y caducifolias) a temperaturas altas que disminuyen la pérdida de agua en la época de sequía. Las especies de leguminosas se restringieron principalmente en las partes bajas de la cuenca. Muchas de estas leguminosas tienen hojas pequeñas (micrófilas) o están transformadas en espinas, lo cual reduce la pérdida de agua por transpiración (Díaz et al., 1998). Otra característica de muchas de las especies de arbustos y árboles encontradas en las partes cálidas de la cuenca es que producen semillas fotoblásticas o termoblásticas, lo que significa que requieren de niveles altos de radiación y temperatura para su germinación $(\mathrm{Ca}-$ margo-Ricalde et al., 2004).

\section{Conclusiones y perspectivas}

La Cuenca de Cuitzeo representa una unidad biogeográfica compleja en términos de diversidad vegetal y características ambientales. Un eje físico que dirige de manera importante la variación ambiental en factores de clima y suelo y, en consecuencia, la distribución de las comunidades y especies vegetales, en la altitud. Dada la notable complejidad ambiental y biológica de la Cuenca de Cuitzeo, es importante considerar las características estructurales y de composición de las comunidades presentes, así como la distribución de las mismas dentro de la cuenca, en el momento de gestionar programas de conservación y manejo. El matorral subtropical, uno de los más diversos tipos de vegetación en la cuenca, está siendo fuertemente impactado por actividades agropecuarias y es importante considerar cuáles prácticas productivas pueden ser compatibles con la conservación de la diversidad vegetal de ese tipo de vegetación. Si bien los bosques de coníferas de la parte alta de la cuenca no presentan una alta diversidad, sí tienen valores altos de biomasa y por lo tanto, tales comunidades vegetales pueden estar desempeñando un papel importante en la captura y almacenamiento de carbono, así como en la protección del suelo y propiedades hidrológicas de la Cuenca de Cuitzeo. Debido a su elevada biomasa, estos bosques están siendo impactados principalmente por actividades de extracción de madera y 
de producción de carbón vegetal, por lo que es fundamental regular dichas actividades para evitar su degradación. Considerando que el bosque mixto tiene una elevada diversidad de especies, una elevada biomasa y una importante extensión en la Cuenca de Cuitzeo, debería ser una prioridad el incluir a este bosque en las políticas de protección de la biodiversidad y de los servicios ecosistémicos aportados por la Cuenca de Cuitzeo.

\section{Agradecimientos}

Agradecemos a Jesús Fuentes Junco de la ENES-Morelia, por la elaboración del mapa del área de estudio y a Rodrigo Velázquez Durán del laboratorio de bioquímica de suelos del CIEco-UNAM por los datos de suelo proporcionados. Lorena Alvarado, Santiago Arizaga-Pérez, Juan Martínez-Cruz, Miguel A. Pérez-Pérez, Sandra Quijas e Ignacio Torres formaron parte de un equipo técnico que ayudó en la colecta de datos de campo. Agradecemos la muy cuidadosa revisión de dos árbitros anónimos que mejoraron de manera importante una versión anterior del presente artículo. Este trabajo fue financiado por el Macro-proyecto "Manejo de Ecosistemas y Desarrollo Humano", de la Universidad Nacional Autónoma de México. Susana Maza Villalobos Méndez agradece la beca posdoctoral otorgada por la DGAPA-UNAM.

\section{Literatura citada}

Ashton M.S., Brokaw N.V.L., Bunyavejchwin R., Chuyong G.B., Co L., Dattaraja H.S., Davies S.J., Esufali S., Ewango C.E.N., Foster R.B., Gunatilleke N., Gunatilleke S., Hart T.H., Hernández C., Hubbell S.P., Itoh A., John R., Kanzaki M., Kenfack D.S.K., LaFrankie J.V., Lee H.S., Liengola I., Makana J.R., Manokaran N., Navarette-Hernández M., Ohkugo T., Perez R., Pongpattananurak N., Samper C., Sri-ngernyuang K., Sukumar R., Fun I.F., Sureh H.S., Tan S., Thomas D.W., Thompson J.D., Vallejo M.I., Villa-Munoz G., Valencia R., Yamakura T. y Zimmerman J.K. 2004. Floristics and vegetation of the Forest Dynamics Plots. En: Losos E.C. y Leigh E.G.Jr. Eds. Tropical Forest Diversity and Dynamism: Findings from a Large-Scale Plot Network, pp. 90102, University of Chicago Press, Chicago.

Balvanera P., Lott E., Segura G., Siebe C. e Islas A. 2002. Patterns of $\beta$-diversity in a Mexican tropical dry forest. 2002. Journal of Vegetation Science 13:145-158.

Bocco G., López-Granados E. y Mendoza M.E. 2012. La investigación ambiental en la cuenca del Lago de Cuitzeo: Una revisión de la bibliografía publicada. En: Bravo-Espinosa M., Barrera-Camacho G., Mendoza M.E., Sáenz J.T., Bahena-Juárez F. y SánchezMartínez R. Eds. Contribuciones para el Desarrollo Sostenible de la Cuenca del Lago de Cuitzeo, Michoacán, pp. 317-345, Instituto Nacional de Investigaciones Forestales, Agrícolas y Pecuarias, Centro de Investigación Regional Pacífico Centro, Campo Experimental Uruapan, Universidad Nacional Autónoma de México, Centro de Investigaciones en Geografía Ambiental, Morelia.

Bravo-Espinosa M., Barrera-Camacho G., Mendoza M.E., Sáenz J.T., Bahena-Juárez F. y Sánchez-Martínez R. 2012. Contribuciones para el Desarrollo Sostenible de la Cuenca del Lago de
Cuitzeo, Michoacán. Instituto Nacional de Investigaciones Forestales, Agrícolas y Pecuarias, Centro de Investigación Regional Pacífico Centro, Campo Experimental Uruapan, Universidad Nacional Autónoma de México, Centro de Investigaciones en Geografía Ambiental. Morelia.

Cabrera González A., Ayala Gómez J.M. y Ortega Rodríguez J.M. 1999. Relación suelo-geoformas-vegetación en la porción sur de la subcuenca de Cuitzeo, Michoacán, México. Ciencia Nicolaita 21:37-53.

Camargo-Ricalde S.L., Dhillion S.S. y García-García V. 2004. Phenology, and seed production and germination of seven endemic Mimosa species (Fabaceae-Mimosoideae) of the Tehuacán-Cuicatlán Valley, Mexico. Journal of Arid Enviroments 58:423-437.

Colwell R.K. 2009. EstimateS v. 8.2.0: Statistical estimation of species richness and shared species from samples. <viceroy. eeb.uconn.edu/EstimateS/> (consultado 20 noviembre 2012).

Colwell R.K., Chao A., Gotelli N.J., Lin S.Y., Mao C.X., Chazdon R.L. y Longino J.T. 2012. Models and estimators linking individual-based and sample-based rarefaction, extrapolation, and comparison of assemblages. Journal of Plant Ecology 5:3-21.

Cué-Bär E.M., Villaseñor J.L., Arredondo-Amezcua L., CornejoTenorio G. e Ibarra-Manríquez G. 2006. La flora arbórea de Michoacán, México. Boletín de la Sociedad Botánica de México 78:47-81.

Díaz S., Cabido M. y Casanoves F. 1998. Plant functional traits and environmental filters at a regional scale. Journal of Vegetation Science 9:113-122.

Encina Domínguez J.A., Zárate Lupercio A., Estrada Castillón E., Valdés Reyna J. y Villareal Quintanilla J.A. 2009. Composición y aspectos estructurales de los bosques de encino de La Sierra de Zapalinamé, Coahuila, México. Acta Botanica Mexicana 86:71-108.

Espinosa D. y Ocegueda S. 2008. El conocimiento biogeográfico de las especies y su regionalización natural. En: Sarukhán J., Soberón J., Halffter G. y Llorente-Bousquets J. Compiladores. Capital Natural de México. Volumen I: Conocimiento Actual de la Biodiversidad, pp. 33-65, Comisión Nacional para el Conocimiento y Uso de la Biodiversidad, México, D.F.

Ferrusquía-Villafranca I. 1993. Geology of Mexico: A synopsis. En: Ramamoorthy T.P., Bye R., Lot A. y Fa J. Eds. Biological Diversity of Mexico. Origins and Distribution, pp. 3-107, Oxford University Press, Nueva York.

García E. 1988. Modificaciones al sistema de clasificación climática de Köppen (para adaptarlo a las condiciones de la República Mexicana). Instituto de Geografía, Universidad Nacional Autónoma de México. México, D.F.

Garduño-Monroy V.H., Corona-Chávez P., Israde-Alcántara I., Menella L., Arreygue E., Bigioggero B. y Chiesa S. 1999. Carta Geológica del Estado de Michoacán, escala 1:250,000: Morelia. Universidad Michoacana de San Nicolás de Hidalgo, Morelia. Morelia.

Gentry A.H. 1982. Patterns of Neotropical plant species diversity. Evolutionary Biology 15:1-84.

Grime J.P. 1982. Estrategias de Adaptación de las Plantas y Procesos que Controlan la Vegetación. Limusa. México, D.F.

INEGI. Instituto Nacional de Estadística, Geografía e Informática. 2005. Guía para la interpretación de cartografía. Uso de suelo y vegetación. Instituto Nacional de Estadística, Geografía e Informática. Aguascalientes. 
Lamont B.B., Le Maitre D.C., Cowling R.M. y Enright N.J. 1991. Canopy seed storage in woody plants. Botanical Review 57:277317.

Leal-Nares O.A., Mendoza M.E. y Carranza González E. 2010. Análisis y modelamiento espacial de información climática en la cuenca de Cuitzeo, México. Investigaciones Geográficas 72:49-67.

Likens G.E., Bormann F.H., Johnson N.M. y Pierce R.S. 1967. The calcium, magnesium, potassium and sodium budgets for a small forested ecosystem. Ecology 48:772-784.

Madrigal Sánchez X. 1995. Ensayo de clasificación de la vegetación con base en el método Tipos de Hábitat. Ciencia Nicolaita 8:49-72.

Madrigal-Sánchez X. y Guridi Gómez L. 2002. Los árboles silvestres del municipio de Morelia, Michoacán, México. Ciencia Nicolaita 33:29-55.

Madrigal Sánchez X. y Trujillo García M.P. 2001. Algunas consideraciones para la planeación de plantaciones en la cuenca de Cuitzeo, Mich. México. Ciencia Nicolaita 27: 45-61.

Magurran A.E. 2004. Measuring Biological Diversity. Blackwell Publishing. Oxford.

Masera D. 2002. Hacia un consumo sustentable. En: Leff E., Ezcurra E., Pisanty I. y Romero L.P. Eds. La Transición hacia el Desarrollo Sustentable. Perspectivas de América Latina y el Caribe, pp. 61-89, Secretaría del Medio Ambiente y Recursos Naturales, Instituto Nacional de Ecología, Universidad Autónoma Metropolitana, Organización de las Naciones Unidas, Programa de las Naciones Unidas para el Medio Ambiente, México D.F.

Mendoza M.E., López E. y Bocco G. 2004. Evaluación del efecto del cambio de cobertura vegetal y uso del suelo en el balance hídrico de grandes cuencas. El caso de la cuenca del Lago de Cuitzeo, Michoacán, México. En: Garduño M.V.H. Ed. Contribuciones a la Geología e Impacto Ambiental de la Región de Morelia, pp. 67-79, Universidad Michoacana de San Nicolás de Hidalgo, Morelia.

Mendoza M.E., Bocco G., López-Granados E. y Bravo E.M. 2010. Hydrological implications of land use and land cover change: Spatial analytical approach at regional scale in the closed basin of the Cuitzeo Lake, Michoacan, Mexico. Singapor Journal of Tropical Geography 31:197-214.

Minchin P.R. 1987. Simulation of multidimensional community patterns: towards a comprehensive model. Vegetatio 71:145156.

PRIMER-E. 2001. Primer v.5.2.9 for Windows. Primer-E Ltd. Plymouth.

Rocha-Loredo A.G. y Ramírez-Marcial N. 2009. Producción y descomposición de hojarasca en diferentes condiciones sucesionales del bosque de pino-encino en Chiapas, México. Boletín de la Sociedad Botánica de México 84:1-12.

Rzedowski J. 1978. Vegetación de México. Limusa. México, D.F.

Rzedowski J. 1991. Diversidad y orígenes de la flora fanerogámica de México. Acta Botanica Mexicana 14:3-21.

Rzedowski J. y Calderón R.G. 1989. Sinopsis numérica de la flora fanerogámica del Valle de México. Acta Botanica Mexicana 8:15-30.

Rzedowski G.C. y Rzedowski J. 2005. Flora Fanerogámica del Valle de México. Comisión Nacional para el Conocimiento y Uso de la Biodiversidad. Pátzcuaro.

Secretaría de la Convención de Ramsar. 2010. Manejo de cuencas hidrográficas: Integración de la conservación y del uso racional de los humedales en el manejo de las cuencas hidrográficas. Manuales Ramsar para el uso racional de los humedales, $4^{a}$ edición, vol. 9. Secretaría de la Convención de Ramsar, Gland.

Smith L.F., Gratz Z.S. y Bousquet S.G. 2009. The Art and Practice of Statistics. Wadsworth.

Strasburger E., Shenck N.H. y Schimper A.F.W. 1960. Tratado de Botánica. Manuel Marian. Barcelona.

Valencia-A. S. 2004. Diversidad del género Quercus (Fagaceae) en México. Boletín de la Sociedad Botánica de México 75:33-53.

Vázquez G.J.A. y Givinish T.J. 1998. Altitudinal gradients in tropical forest composition, structure, and diversity in the Sierra de Manantlán. Journal of Ecology 86:999-1020.

Villaseñor J.L. 1993. La familia Asteraceae en México. Revista de la Sociedad Mexicana de Historia Natural 44:117-124.

Villaseñor J.L. 2003. Diversidad y distribución de las Magnoliophyta de México. Interciencia 28:160-167.

Recibido: 3 de julio de 2013

Aceptado: 22 de agosto de 2013 
Apéndice 1. Lista de especies de árboles y arbustos $(1 \geq \mathrm{cm}$, DAP) registrados en cinco tipos de vegetación presentes en la Cuenca de Cuitzeo. En orden de altitud creciente los tipos de vegetación fueron: MS, matorral subtropical; BE, bosque de encino; $\mathrm{BM}$, bosque mixto; $\mathrm{BP}$, bosque de pino; $\mathrm{BO}$, bosque de oyamel.

\begin{tabular}{|c|c|c|c|c|c|c|}
\hline Familia & Especie & MS & BE & BM & BP & BO \\
\hline \multirow[t]{2}{*}{ ANACARDIACEAE } & Schinus molle L. & $x$ & & & & \\
\hline & Pistacia mexicana H.B.K. & $x$ & $x$ & & & \\
\hline APOCYNACEAE & Plumeria sp. & $x$ & & & & \\
\hline AQUIFOLIACEAE & Ilex sp. & & & $x$ & & \\
\hline ARALIACEAE & Dendropanax sp. & & & $\mathrm{x}$ & & \\
\hline \multirow[t]{14}{*}{ ASTERACEAE } & Ageratina sp. & & & $\mathrm{x}$ & & \\
\hline & sp. 1 & $\mathrm{x}$ & & & & \\
\hline & sp. 2 & & $x$ & & & \\
\hline & sp. 3 & $x$ & & $x$ & & \\
\hline & sp. 4 & $\mathrm{x}$ & & & & \\
\hline & sp. 5 & $x$ & & & & \\
\hline & sp. 6 & $x$ & & & & \\
\hline & sp. 7 & $\mathrm{x}$ & & & & \\
\hline & sp. 8 & $\mathrm{x}$ & & & & \\
\hline & sp. 9 & $x$ & & & & \\
\hline & Baccharis conferta Kunth & $x$ & $x$ & & & \\
\hline & Baccharis heterophylla Kunth & $x$ & $x$ & $x$ & $\mathrm{x}$ & $x$ \\
\hline & Baccharis pteronioides DC. & $x$ & & & & \\
\hline & Eupatorium glabratum Kunth & & & $x$ & & \\
\hline \multirow[t]{16}{*}{ ASTERACEAE } & Eupatorium sp. 1 & $x$ & $x$ & $x$ & & \\
\hline & Eupatorium sp. 2 & $\mathrm{x}$ & & $\mathrm{x}$ & & \\
\hline & Eupatorium sp. 3 & $x$ & & & & \\
\hline & Montanoa grandiflora DC. & $\mathrm{x}$ & & $\mathrm{x}$ & & \\
\hline & Montanoa tomentosa Cerv. & $\mathrm{x}$ & $\mathrm{x}$ & & & \\
\hline & Montanoa sp. & & $\mathrm{x}$ & & & \\
\hline & Senecio cinerarioides Kunth & $x$ & & & & \\
\hline & Senecio salignus DC. & & & & & $\mathrm{x}$ \\
\hline & Verbesina alata $\mathrm{L}$. & $\mathrm{x}$ & $\mathrm{x}$ & & & \\
\hline & Verbesina oligantha B.L.Rob & $x$ & $x$ & $\mathrm{x}$ & & \\
\hline & Verbesina oncophora B.L.Rob. \& Seaton & $x$ & & & & \\
\hline & Verbesina sp. 1 & $x$ & & $x$ & & \\
\hline & Verbesina sp. 2 & $\mathrm{x}$ & & & & \\
\hline & Vernonia paniculata DC. & $\mathrm{x}$ & & & & \\
\hline & Vernonia sp. 1 & $\mathrm{x}$ & & & & \\
\hline & Vigethia sp. 2 & $\mathrm{x}$ & & & & \\
\hline BEGONIACEAE & Begonia sp. & $\mathrm{x}$ & & & & \\
\hline BERBERIDACEAE & Berberis moranensis Schult. \& Schult.F. & & & & $\mathrm{x}$ & \\
\hline BETULACEAE & Alnus acuminata Kunth & & & $x$ & & $x$ \\
\hline BIGNONIACEAE & Tecoma stans (L.) Kunth & $\mathrm{x}$ & & & & \\
\hline \multirow[t]{2}{*}{ BOMBACACEAE } & Ceiba aesculifolia (Kunth) Britt. \& Baker f. & $\mathrm{x}$ & & & & \\
\hline & Ceiba sp. & $x$ & & & & \\
\hline BORAGINACEAE & Cordia sp. & $\mathrm{x}$ & & & & \\
\hline \multirow[t]{2}{*}{ BORAGINACEAE } & Ehretia latifolia Loisel. & $\mathrm{x}$ & & & & \\
\hline & sp. 10 & $\mathrm{x}$ & & & & \\
\hline \multirow[t]{4}{*}{ BURSERACEAE } & Bursera citronella McVaugh \& Rzed. & $\mathrm{x}$ & & & & \\
\hline & Bursera cuneata Engl. & $x$ & $\mathrm{x}$ & & & \\
\hline & Bursera excelsa Engl. & $\mathrm{x}$ & & & & \\
\hline & Bursera fagaroides (Kunth) Engl. & $\mathrm{x}$ & $\mathrm{x}$ & & & \\
\hline
\end{tabular}


Apéndice 1. Continuación

\begin{tabular}{|c|c|c|c|c|c|c|}
\hline Familia & Especie & MS & BE & BM & $\mathbf{B P}$ & BO \\
\hline & Bursera microphylla A.Gray & $x$ & & & & \\
\hline & Bursera sp. & $x$ & & & & \\
\hline CAPRIFOLIACEAE & Viburnum acutifolium Benth. & $x$ & & & & \\
\hline CASUARINACEAE & Casuarina equisetifolia J.R.Forst. \& G.Forst. & & & $\mathrm{x}$ & & \\
\hline CLETHRACEAE & Clethra sp. & & & $x$ & $x$ & \\
\hline \multirow[t]{2}{*}{ CONVOLVULACEAE } & Ipomoea arborescens Sweet & $x$ & $x$ & $x$ & & \\
\hline & Ipomea murucoides Roem. \& Schult. & $x$ & $x$ & $x$ & & \\
\hline CORNACEAE & Cornus sp. & & & $x$ & & \\
\hline \multirow[t]{3}{*}{ ERICACEAE } & Arbutus glandulosa M.Martens \& Galeotti. & & & $x$ & & \\
\hline & Arbutus tessellata P.D.Sorensen & & & $x$ & & \\
\hline & Arbutus xalapensis Kunth. & & $x$ & $x$ & & \\
\hline \multirow[t]{3}{*}{ EUPHORBIACEAE } & Croton sp. 1 & $\mathrm{x}$ & $\mathrm{x}$ & & & \\
\hline & Croton sp. 2 & $\mathrm{x}$ & $\mathrm{x}$ & & & \\
\hline & Jatropha sp. & $x$ & & & & \\
\hline \multirow[t]{3}{*}{ FABACEAE } & Acacia cochliacantha Humb. \& Bonpl. Ex Willd. & $\mathrm{x}$ & & & & \\
\hline & Acacia farnesiana (L.) Wild. & $x$ & $x$ & & & \\
\hline & Acacia pennatula (Schltdl. \& Cham.) Benth. & $x$ & $x$ & & & \\
\hline \multirow[t]{13}{*}{ FABACEAE } & Albizia occidentalis Brandegee & $\mathrm{x}$ & & & & \\
\hline & Albizia sp. & $x$ & & & & \\
\hline & Calliandra grandiflora (L'Her.) Benth. & $\mathrm{x}$ & $\mathrm{x}$ & $\mathrm{x}$ & & \\
\hline & Calliandra sp. & $x$ & & & & \\
\hline & Erythrina americana Mill. & $x$ & & & & \\
\hline & Eysenhardtia polystachya (Ortega) Sarg. & $x$ & $\mathrm{x}$ & & & \\
\hline & Lysiloma sp. & $x$ & & & & \\
\hline & Mimosa sp. & $\mathrm{x}$ & $x$ & & & \\
\hline & Prosopis laevigata (H. \& B.) Jonhst & $\mathrm{x}$ & & & & \\
\hline & sp. 10 & $\mathrm{x}$ & & & & \\
\hline & sp. 11 & $x$ & & & & \\
\hline & sp. 12 & $\mathrm{x}$ & & & & \\
\hline & sp. 13 & $x$ & & & & \\
\hline \multirow[t]{10}{*}{ FAGACEAE } & Quercus acutifolia Née. & & & $\mathrm{x}$ & & \\
\hline & Quercus affinis M.Martens \& Galeotti & & & $x$ & & \\
\hline & Quercus candicans Née & $\mathrm{x}$ & $\mathrm{x}$ & $\mathrm{x}$ & & \\
\hline & Quercus castanea Née & $x$ & $x$ & $x$ & & \\
\hline & Quercus conspersa Benth. & & & $\mathrm{x}$ & & \\
\hline & Quercus crassifolia Humb. \& Bonpl. & & $\mathrm{x}$ & $\mathrm{x}$ & & \\
\hline & Quercus crassipes Humb. \& Bonpl. & & $x$ & $x$ & & \\
\hline & Quercus deserticola Trel. & $x$ & $\mathrm{x}$ & $\mathrm{x}$ & & \\
\hline & Quercus gentryi C.H. Mull. & & & $\mathrm{x}$ & & \\
\hline & Quercus glabrescens Benth. & & & $x$ & & \\
\hline \multirow[t]{7}{*}{ FAGACEAE } & Quercus glaucoides M.Martens \& Galeotti & $\mathrm{x}$ & $\mathrm{x}$ & & & \\
\hline & Quercus laeta Liebm. & $\mathrm{x}$ & $\mathrm{x}$ & $\mathrm{x}$ & & \\
\hline & Quercus laurina M.Martens \& Galeotti & & & & & $\mathrm{x}$ \\
\hline & Quercus obtusata Humb. \& Bonpl. & $\mathrm{x}$ & $\mathrm{x}$ & $\mathrm{x}$ & & \\
\hline & Quercus peduncularis Née. & $x$ & $x$ & $x$ & & \\
\hline & Quercus resinosa Liebm. & $\mathrm{x}$ & & $\mathrm{x}$ & & \\
\hline & Quercus rugosa Née. & & & $\mathrm{x}$ & $\mathrm{x}$ & \\
\hline GARRYACEAE & Garrya laurifolia Benth. & $x$ & $x$ & & & \\
\hline HYDROPHYLLACEAE & Wigandia urens (Ruiz \& Pavón) H.B.K. & & & $\mathrm{x}$ & & \\
\hline LAMIACEAE & Asterohyptis sp. & $x$ & & & & \\
\hline
\end{tabular}


Apéndice 1. Continuación

\begin{tabular}{|c|c|c|c|c|c|c|}
\hline Familia & Especie & MS & BE & BM & $\mathbf{B P}$ & BO \\
\hline & Salvia sp. 1 & & $x$ & $x$ & & \\
\hline & Salvia sp. 2 & & $x$ & & & \\
\hline LOGANIACEAE & Buddleja sp. & $x$ & & $x$ & & \\
\hline \multirow[t]{2}{*}{ MALPIGHIACEAE } & Galphimia glauca Cav. & $x$ & & & & \\
\hline & 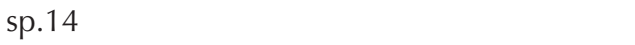 & $x$ & & & & \\
\hline MELIACEAE & Cedrela dugesii S.Watson & $x$ & & & & \\
\hline MYRTACEAE & Eucalyptus sp. & $x$ & & & & \\
\hline \multirow[t]{2}{*}{ OLEACEAE } & Forestiera phillyreoides Torr. & $\mathrm{x}$ & $\mathrm{x}$ & & & \\
\hline & Fraxinus uhdei (Wenzig) Lingelsh. & $\mathrm{x}$ & & $x$ & & \\
\hline OPILIACEAE & Agonandra racemosa Standl. & $x$ & & & & \\
\hline \multirow{3}{*}{ PINACEAE } & Abies religiosa (Kunth) Schltdl. \& Cham. & & & $x$ & $x$ & $x$ \\
\hline & Pinus ayacahuite Ehrenb. ex Schlecht. & & & $x$ & & \\
\hline & Pinus herrerai Martínez & & & $x$ & & \\
\hline \multirow[t]{8}{*}{ PINACEAE } & Pinus leiophylla Schltdl. \& Cham. & & & $x$ & & \\
\hline & Pinus michoacana Martínez & & & $x$ & & \\
\hline & Pinus montezumae Lamb. & & & $x$ & & \\
\hline & Pinus oocarpa Schiede ex Schltdl & & & $x$ & $x$ & \\
\hline & Pinus pringlei Shaw & & & $x$ & & \\
\hline & Pinus pseudostrobus Lindl. & $x$ & & & & \\
\hline & Pinus teocote Cham. \& Schltdl. & $x$ & $x$ & $x$ & & \\
\hline & Pinus sp. & & & $x$ & $x$ & \\
\hline \multirow[t]{3}{*}{ RHAMNACEAE } & Condalia mexicana Schltdl. & & & $x$ & & \\
\hline & Condalia velutina I.M.Johnston & $x$ & $\mathrm{x}$ & & & \\
\hline & Condalia sp. & & $x$ & & & \\
\hline \multirow[t]{8}{*}{ ROSACEAE } & Amelanchier denticulata (Kunth) K.Koch & $\mathrm{x}$ & $\mathrm{x}$ & & & \\
\hline & Crataegus mexicana Moc. \& Sessé ex DC. & $\mathrm{x}$ & $\mathrm{x}$ & $x$ & $x$ & \\
\hline & Crataegus sp. & $x$ & & & & \\
\hline & Prunus serotina subsp. capuli (Cav. ex Spreng) & $x$ & & $\mathrm{x}$ & & \\
\hline & McVaugh & & & & & \\
\hline & Prunus sp. & & & $x$ & & \\
\hline & sp. 15 & $\mathrm{x}$ & & & & \\
\hline & sp. 16 & $x$ & & & & \\
\hline \multirow[t]{3}{*}{ RUBIACEAE } & Bouvardia sp. & $x$ & & & & \\
\hline & Randia sp. & $x$ & & & & \\
\hline & sp. 17 & $x$ & & & & \\
\hline \multirow[t]{2}{*}{ RUTACEAE } & Casimiroa edulis La Llave & $x$ & & & & \\
\hline & Zanthoxylum fagara Sarg. & $x$ & & & & \\
\hline SALICACEAE & Salix sp. & & & $x$ & & $x$ \\
\hline SAPINDACEAE & Dodonaea viscosa Jacq. & $x$ & & & & \\
\hline \multirow[t]{4}{*}{ SOLANACEAE } & Cestrum fulvescens Fernald & $x$ & & & & \\
\hline & Cestrum sp. & $x$ & $x$ & & & \\
\hline & Solanum cervantesii Lag. & $x$ & & & & \\
\hline & Solanum sp. & $\mathrm{x}$ & & & & \\
\hline STYRACACEAE & Styrax argenteus C.Presl & & & $x$ & & \\
\hline SYMPLOCACEAE & Symplocos citrea Lex. & & & $\mathrm{x}$ & & \\
\hline \multirow[t]{3}{*}{ THEACEAE } & Ternstroemia lineata DC. & & & $\mathrm{x}$ & & \\
\hline & Ternstroemia pringlei Standl. & & & $x$ & & \\
\hline & Ternstroemia sp. & & & & $x$ & \\
\hline \multirow[t]{2}{*}{ TILIACEAE } & Heliocarpus sp. & $x$ & & & & \\
\hline & sp. 18 & $x$ & & & & \\
\hline
\end{tabular}


Apéndice 1. Continuación

\begin{tabular}{|c|c|c|c|c|c|c|}
\hline Familia & Especie & MS & BE & BM & BP & BO \\
\hline \multirow[t]{2}{*}{ ULMACEAE } & Celtsi sp. & $x$ & & $\mathrm{x}$ & & \\
\hline & sp. 19 & $x$ & & & & \\
\hline \multirow[t]{5}{*}{ VERBENACEAE } & Lantana camara L. & $x$ & & & & \\
\hline & Lippia mexicana G.L.Nesom. & $x$ & & & & \\
\hline & Lippia umbellata Cav. & $\mathrm{x}$ & & & & \\
\hline & Lippia sp. & $x$ & $x$ & & & \\
\hline & sp. 20 & $x$ & & & & \\
\hline \multirow[t]{3}{*}{ MORFOESPECIES } & Morfoespecie 1 & & & $\mathrm{x}$ & & \\
\hline & Morfoespecie 2 & & & $\mathrm{x}$ & & \\
\hline & Morfoespecie 3 & & & $\mathrm{x}$ & & \\
\hline \multirow[t]{10}{*}{ MORFOESPECIES } & Morfoespecie 4 & & & $\mathrm{x}$ & & \\
\hline & Morfoespecie 5 & $x$ & & & & \\
\hline & Morfoespecie 6 & $x$ & & & & \\
\hline & Morfoespecie 7 & $x$ & & & & \\
\hline & Morfoespecie 8 & $x$ & & & & \\
\hline & Morfoespecie 9 & $x$ & & & & \\
\hline & Morfoespecie 10 & & & $\mathrm{x}$ & & \\
\hline & Morfoespecie 11 & & $\mathrm{x}$ & & & \\
\hline & Morfoespecie 12 & & & $x$ & & \\
\hline & Morfoespecie 13 & & $\mathrm{x}$ & & & \\
\hline
\end{tabular}

